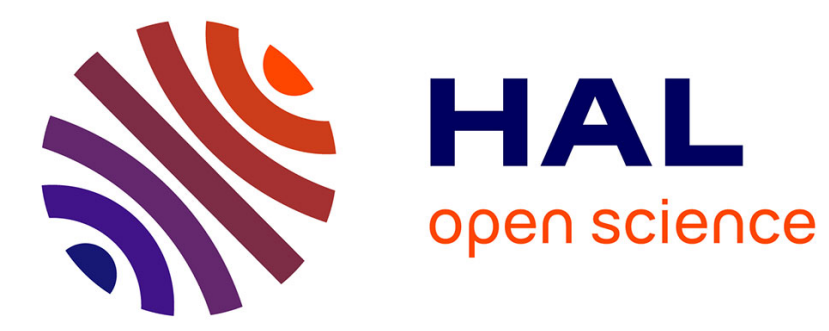

\title{
Turning an Articulated 3-PPSR Manipulator into a Parallel Continuum Robot
}

Oscar Gallardo, Benjamin Mauze, Redwan Dahmouche, Christian Duriez, Guillaume Laurent

\section{- To cite this version:}

Oscar Gallardo, Benjamin Mauze, Redwan Dahmouche, Christian Duriez, Guillaume Laurent. Turning an Articulated 3-PPSR Manipulator into a Parallel Continuum Robot. IROS 2021 - IEEE/RSJ International Conference on Intelligent Robots and Systems, Sep 2021, Prague, Czech Republic. 10.1109/IROS51168.2021.9636596 . hal-03549322

\section{HAL Id: hal-03549322 \\ https://hal.science/hal-03549322}

Submitted on 31 Jan 2022

HAL is a multi-disciplinary open access archive for the deposit and dissemination of scientific research documents, whether they are published or not. The documents may come from teaching and research institutions in France or abroad, or from public or private research centers.
L'archive ouverte pluridisciplinaire HAL, est destinée au dépôt et à la diffusion de documents scientifiques de niveau recherche, publiés ou non, émanant des établissements d'enseignement et de recherche français ou étrangers, des laboratoires publics ou privés. 


\title{
Turning an Articulated 3-PPSR Manipulator into a Parallel Continuum Robot
}

\author{
Oscar F. Gallardo, Benjamin Mauzé, Redwan Dahmouche, \\ Christian Duriez, and Guillaume J. Laurent
}

\begin{abstract}
Parallel Continuum Robots (PCR) have received a lot of attention in recent years. This paper presents a new 6-degrees-of-freedom PCR derived from the conventional 3PPSR parallel manipulator. This robot is driven by three limbs consisting of two flexible rods each and replacing the spherical and revolute joints of the original version. Each limb is mounted onto two linear axes arranged in series. To allow a direct comparison between the articulated and the continuum version, the parallel mechanism of an industrial manipulator has been replaced by an elastic structure of the same size. The simulations and the experiments show that the flexible counterpart of the manipulator is able to achieve a larger workspace, increasing the range of motion by $150 \%$ for rotations and by $157 \%$ in elevation. Moreover, the position repeatability is improved by $47 \%$ (reaching $3.4 \mu \mathrm{m}$ ) and the orientation repeatability by $57 \%$ (reaching $14.3 \mu \mathrm{rad}$ ). This can be explained by the removal of the spherical and revolute joints but also by the constant stress in the structure that acts as an anti backlash system on leadscrew actuators.
\end{abstract}

\section{INTRODUCTION}

Conventional articulated robots have been studied for decades in terms of modeling, design and control to address many requirements such as speed, size, repeatability, workspace, dexterity, and safe human/robot interactions.

To fulfill the need for high repeatability, parallel kinematics has been one of the most considered robot structures because they provides high ratios of rigidity over weight and shorter kinematic chains. In 2014, the concept of parallel continuum robots (PCR) was introduced by Bryson \& Rucker [1]. This concept opened new applications for parallel structures by compensating the loss of workspace and dexterity that rigid-link parallel robots suffer from. PCR consist of flexible limbs linked in parallel to a rigid mobile platform. They have some similarities with parallel soft robots introduced previously by Duriez [2], even though they embed some rigid elements to acquire properties suitable in some applications such as medical robotics [3], haptic interfacing [4], collaborative robotics [5] and precision positioning [6].

This work was supported by the $\mu$ Spider project (ANR-19-CE33-000202), by Région Bourgogne Franche-Comté and by the EIPHI Graduate School (ANR-17-EURE-0002). The encoded target has been realized thanks to the RENATECH technological network and its FEMTO-ST facility MIMENTO. Authors acknowledge S. Carbillet and the AMETISTE facility for tensile tests.

Oscar F. Gallardo, Benjamin Mauzé, Redwan Dahmouche, and Guillaume J. Laurent are with FEMTO-ST Institute, Univ. Bourgogne FrancheComté, UMR CNRS 6174, Besançon, France (corresponding author: guillaume.laurent@ens2m.fr)

Christian Duriez is with DEFROST team at INRIA Lille-Nord-Europe, Université de Lille, France

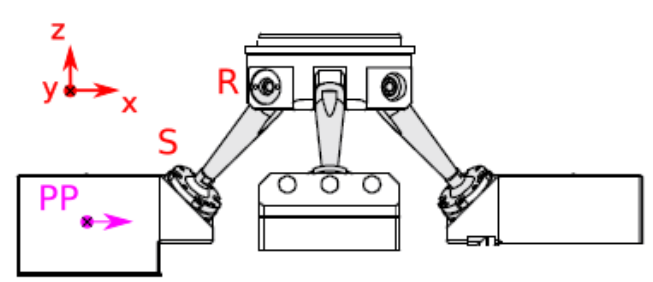

(a) Sketch of the 3-PPSR developed by PI-MICOS

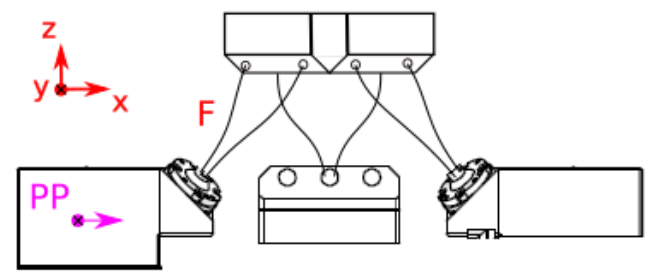

(b) Sketch of the proposed PCR

Fig. 1. Transformation of a conventional 3-PPSR into a parallel continuum robot $(\mathrm{PCR})$.

Studies about classical robotic structures, like the GoughStewart platform, using elastic elements instead of conventional joints is a rising trend. For instance in [7], Black et al. used a Gough-Stewart-like platform for which they developed the forward kinematic model, performed a kinetostatic analysis of the structure and analyzed the pros and cons of PCR. Young and Kuchenbecker [4] used the same 6-degrees-of-freedom (DoF) structure for a fingertip haptic interface. Till et al. demonstrated the possibility of modeling and controlling the robot in quasi-static [8] and dynamic [9] modes using Cosserat rod models.

Planar parallel architectures have also been explored as well with a higher focus on stability and precision. In [10], Altuzarra et al. analyzed the stable and unstable poses of a 2 DoF planar PCR. In 2020, our team introduced a continuum version of the planar 3-PRR parallel robot and demonstrated experimentally a repeatability of few nanometers over a millimetric workspace, paving the way to high-precision positioning PCR [6], [11]. Recently, another continuum counterpart of the 3 - $\underline{R} R R$ was proposed by [12] using tendon-actuated legs.

This paper presents the transformation of the conventional 3-PPSR manipulator into a parallel continuum robot. The 3 PPSR kinematics was introduced by Tahmasebi and Tsai [13] in 1994 and is composed of three serial chains connecting the 
end platform to the base. Each chain consists of two actuated prismatic joints, one spherical joint and one revolute joint at the end. Thus, the resulting robot has 6-DoF at the endeffector. This structure was also studied by Majid and Huang [14] demonstrating that it has a much larger workspace than the Gough-Stewart platform.

Replacing parallel robots' links with flexible limbs would allow to significantly reduce the robots' dimensions to obtain highly miniaturized robots that can be used in medical robotics and micro/nano-manipulation. However, the impact of such a transformation on the robot's performance has to be considered. This paper propose to study the impact of transforming a industrial 3-PPSR parallel robot (illustrated in Fig. 1) into a continuum one and compare three key performances, namely the workspace, the stiffness and the repeatability, .

The study begins with the design and the mechanical sizing of the parallel continuum robot that uses the same actuation system than the industrial robot. Then, the obtained PCR is modeled using finite element modeling to estimate its workspace. Finally, the performance of a functional prototype are evaluated experimentally.

\section{PCR TRANSFORMATION}

The study is based on an existing 3-PPSR industrial robot, the spaceFAB 3000 BS, developed by PI-MICOS. Fig. 1(a) and Fig. 1(b) illustrate respectively the spaceFAB sketch with the location of the joints and the proposed architecture that shows the location of the flexible components denoted by " $F$ ". The transformation from a parallel robot to a PCR can be seen as a succession of two elementary operations. First, the articulated joints are converted to the equivalent compliant joints (flexure hinges [15]), and then the compliant joints are replaced by flexible rods.

A flexure hinge provides the relative rotational motion between two adjacent rigid components via material deformation instead of the sliding or rolling of articulated joints. Flexure hinges are thus characterized by their light weight, the absence of backlash and friction, and the fact that no assembly is usually required. They can be classified as single-axis, two-axis and multi-axis joints [16].

Considering Fig. 1(a), the first transformation is located at the base on the spherical joint $S$. This joint is shown in Fig. 2(a) and it provides 3-DoF (rotation about each axis). So a hourglass hinge is implemented to keep the provided DoF, leading to a compliant spherical equivalence (Fig. 2(b)). Then with a single flexible rod transformation (Fig. 2(c)) we can obtain a similar flexibility for the three rotations if the material of the rod has the mechanical characteristic of high grade bending but negligible compression.

A second transformation is located at the top of the platform, where the revolute joint $R$ is located. In this case, a single-axis flexible hinge is implemented to convert it (Fig. 2(d)) into the notch compliant joint or leaf spring shown in Fig. 2(e). Then, a simplification of the leaf spring into a two parallel flexible rods results into a preferential bending around their common transverse axis (Fig. 2(f)). Additional a) Rigid spherical joint

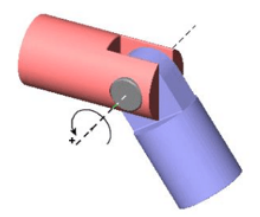

d) Rigid revolute joint

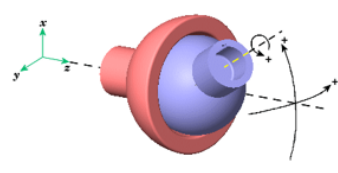

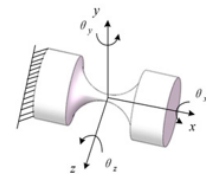

b) Compliant spherical joint

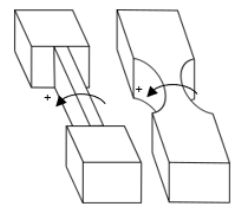

e) Leaf spring and single notch compliant joint

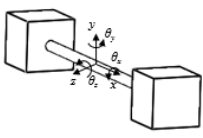
flexible joint

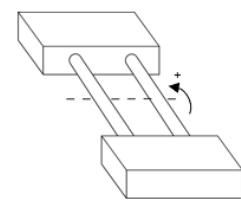

f) Double beam flexible joint c) Single beam

Fig. 2. Transition from articulated joint to flexible joint.

rods can be put diagonally in the same plane to increase the stiffness of other DoF.

Applying both modifications, we substitute each articulated limb by a pair of flexible rods. Those two rods are: clamped close enough at the base to perform as a single rod, and separated at the top to behave as the leaf spring (Fig. 1(b)). Thus, the resulting PCR design is denoted 3PP2-F, each limb composed by two active prismatic joints in series, and two flexible rods in parallel.

\section{MEChANICAL SIZING}

To ensure an equivalent transformation and a fair comparison between the spaceFAB and the proposed PCR, we consider four frames and six geometrical parameters to define the robot. First, the main frame $P$ is placed at the centre of the platform and three supplementary frames $Q_{i}$ (i from 1 to 3 , corresponding to each actuator) are located at the origin of the spherical axes. Fig. 3 illustrates the location of the frames and the six parameters.

Taking this into consideration, two equilateral triangles are considered. One formed between the revolute axes at the platform and the second between the spherical axes at the base for the initial position of the three actuators. Then $r_{p}$ is the radius of the first triangle, measured from the frame $P$ to the centre of one of the sides, and $r_{b}$ is the radius of the second, measured from one of $Q_{i}$ to the projection of the frame $P$ into the second triangle. $h$ is the height between the base and the platform.

In order to keep the equivalence between one articulated limb and two flexible rods, three additional parameters are defined. $g_{p}$ is the distance between clamp fixings of rods at the platform. $g_{b}$ is the distance between clamp fixings of rods at the base. Finally, $l$ is the length of flexible rods.

The dimensional parameters not related to the rods are defined with the same value of the spaceFAB. This way, the proposed PCR will be a closer flexible counterpart of the articulated one. Table I shows the previously mentioned parameters required to completely define the model. The two first parameters $r_{p}$ and $r_{b}$ were obtained directly from the original spaceFAB. We use the tools provided by SOFA (presented in the next section) to model the manipulator 


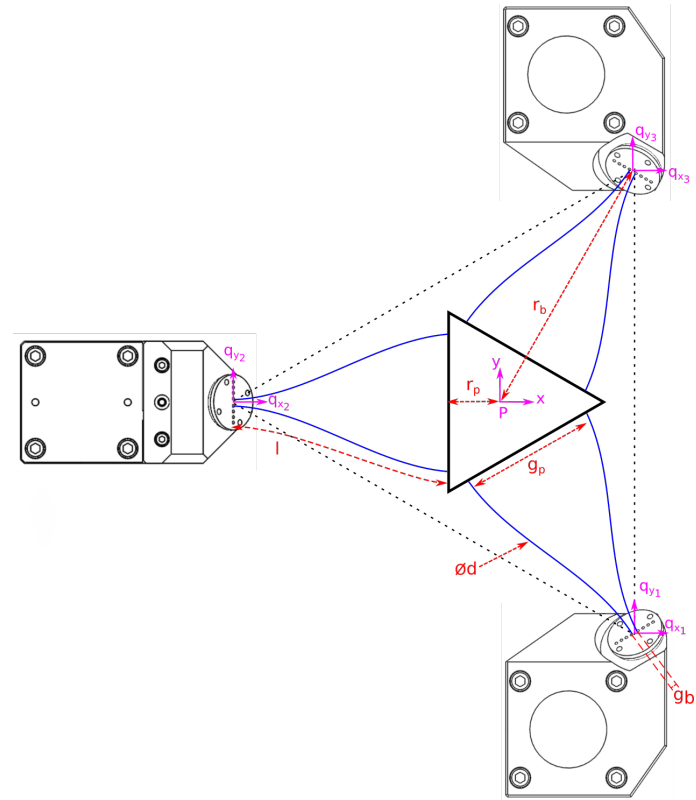

(a) Top view

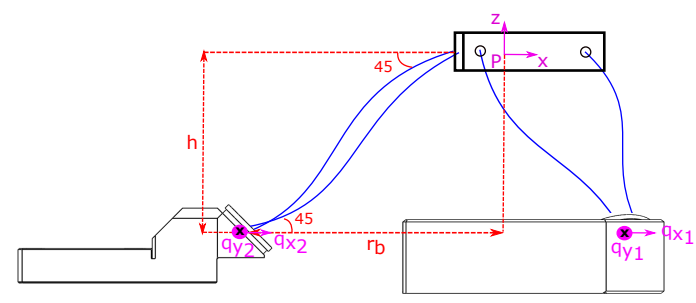

(b) Side view

Fig. 3. Schematic model of the 3-PP2-F.

TABLE I

DIMENSIONAL PARAMETERS OF THE 3-PP2-F.

\begin{tabular}{ccc}
\hline Name & Description & Nominal Value \\
\hline$r_{p}$ & Platform radius & $12.46 \mathrm{~mm}$ \\
\hline$r_{b}$ & Base radius & $74.93 \mathrm{~mm}$ \\
\hline$g_{p}$ & Platform gap & $68.00 \mathrm{~mm}$ \\
\hline$g_{b}$ & Base gap & $4.00 \mathrm{~mm}$ \\
\hline$l$ & Rod length & $79.00 \mathrm{~mm}$ \\
\hline
\end{tabular}

and to define the rod length that elevates the platform to $50.00 \mathrm{~mm}$ as for original spaceFAB.

To ensure the expected behavior, the flexible rods should allow high grade of bending but negligible elongation. For this reason, 1-mm-in-diameter rods made of stainless steel were chosen. Table II gathers the main mechanical properties and dimensions that have been considered in the modeling in the next section.

\section{Modeling And Simulation}

A mechanical model of the robot has been implemented with the open-source framework SOFA [17] along with its plugin SoftRobots [18]. This software provides specific tools to simulate and control continuum and soft robots. The
TABLE II

ROD DIMENSIONS AND PROPERTIES

\begin{tabular}{cc}
\hline Properties & Nominal Value \\
\hline Young's modulus & $160 \mathrm{GPa}$ \\
\hline Poisson's ratio & 0.3 \\
\hline Density & $8000 \mathrm{~kg} \cdot \mathrm{m}^{-3}$ \\
\hline Diameter & $1.00 \pm 0.01 \mathrm{~mm}$ \\
\hline
\end{tabular}

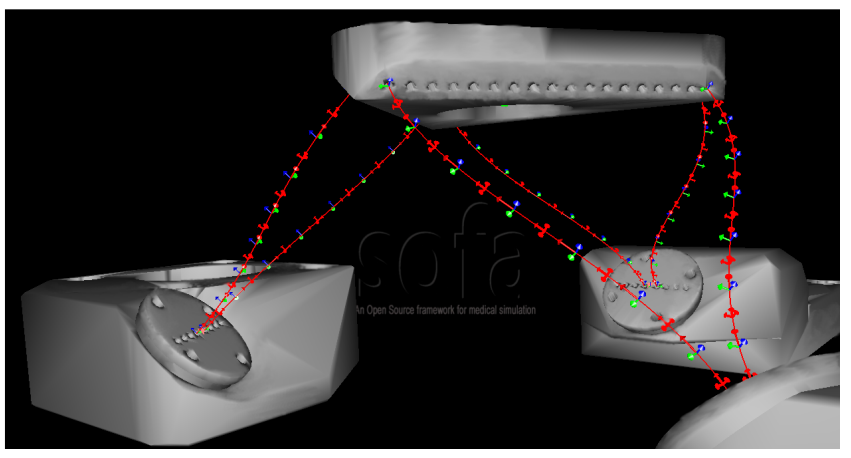

Fig. 4. View of the FEM model of the robot implemented with SOFA. Each rod is discretised in 10 elements.

modeling of flexible bodies is based on 3D finite element models (FEM) with various geometries like beams, shells and solids.

Given the length $(79 \mathrm{~mm})$ of the flexible rods with regards to their diameter (1 mm), 3-D models of beam elements have been considered. The beam elements have a constant circular section and a straight initial shape. Cubic spline curves are used to get a precise interpolation along their main axis. The stiffness matrix $\mathbf{K}$ for the beam elements is obtained by integrating a linear elastic law on the interpolated shape. This $12 \times 12$ matrix links the internal forces and moments $\mathbf{f}_{b}$ applied to the ends of the element with their relative displacements $\mathbf{u}_{b}$ in the local frame of the beam, such as,

$$
\mathbf{f}_{b}=\mathbf{K} \cdot \mathbf{u}_{b}
$$

In SOFA, beam elements take into account one direction of stretching, two directions of bending, one direction of torsion, and two directions of shearing. More details about the model implemented in SOFA can be found in [19].

To handle large deformations, several beam elements must be put together to model each rod. These meshes are then assembled to the actuators and to the platform by applying the rigid transformations corresponding to the geometry given in Fig. 3. The resulting model implemented with SOFA is illustrated in Fig. 4.

\section{A. Number of elements selection}

In finite element analyses, it is known that increasing the number of elements, improves accuracy of the analysis. However, more elements means a longer execution time which is not desirable to achieve real-time control. Hence, it is advised to use the minimum number of elements allowing to reach a sufficient accuracy. 


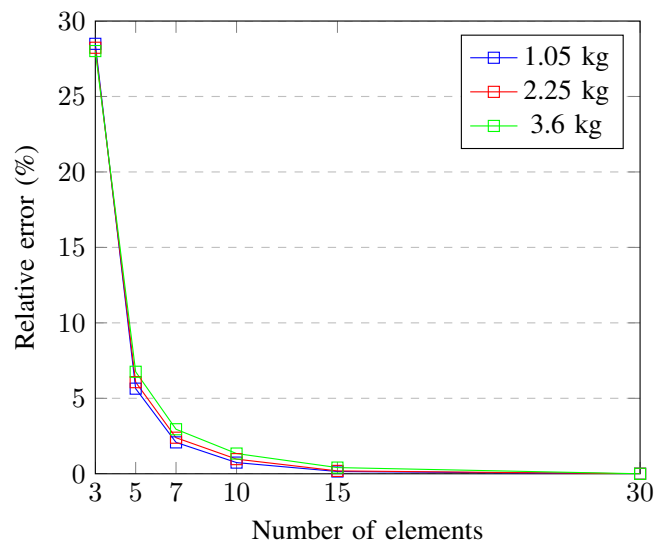

Fig. 5. Element number vs accuracy for different loads.

To find this number, we carried out a static analysis on the vertical stiffness of the robot at its reference configuration. Different loads were applied at the middle of the platform while its elevation was recorded. A series of robot models were generated whose rods have from 1 to 30 beam elements (Fig. 5). In that graph, it is assumed that the model with the highest number of elements generated the most accurate results, being the one with 30 elements. Then the percentage approximate error was calculated by comparing the rest of the results with this one. It can be seen that, the model with fewer number of elements could not correctly reflect the real displacement of the platform. The relative error drops below $1.4 \%$ for 10 elements. Thus, 10 elements per rod are enough to accurately simulate the displacement under constant load.

\section{B. Workspace evaluation}

Taking in consideration the dimension parameters established in Table I, the previous model with 10 elements per rod and the inverse kinematics (IK) plugin provided by SOFA, it is possible to simulate the workspace of the PCR. Indeed, the IK plugin allows to determine the position of the actuators at the base that move the platform to a given pose. So the procedure starts with the platform in the reference position. Then, a small translation (T) or rotation (R) is effected on the platform until one of the actuators reaches its maximal displacement allowed $(+/-25 \mathrm{~mm}$ in $\mathrm{X}$ and $+/-50 \mathrm{~mm}$ in $\mathrm{Y})$. The same procedure is done for the opposite side and for all the directions. This way the workspace is obtained in terms of the maximal displacements of the platform along the major directions.

The results are shown in the Table III and compared to those of the spaceFAB. We can observe that the translations Tx and Ty are identical in both robots. This value corresponds to the maximal displacements of the actuators because the whole robot moves together when effecting them. But, for the translation $\mathrm{Tz}$, it is quite different. On one hand, the PCR can travel in a larger range. This improvement is due to the large bending allowed by the rods. On the other hand, the spaceFAB is limited by the mechanical stops of the spherical joints at the base. This same improvement is
TABLE III

COMPARISON OF THE WORKSPACE BETWEEN THE ORIGINAL ARTICULATED ROBOT SPACEFAB AND THE PCR.

\begin{tabular}{ccccrrr}
\hline & $\begin{array}{c}\text { Tx } \\
(\mathrm{mm})\end{array}$ & $\begin{array}{c}\text { Ty } \\
(\mathrm{mm})\end{array}$ & $\begin{array}{c}\text { Tz } \\
(\mathrm{mm})\end{array}$ & $\begin{array}{r}\mathbf{R x} \\
\left({ }^{\circ}\right)\end{array}$ & $\begin{array}{r}\mathbf{R y} \\
\left({ }^{\circ}\right)\end{array}$ & $\begin{array}{c}\mathbf{R z} \\
\left({ }^{\circ}\right)\end{array}$ \\
\hline \multirow{2}{*}{ spaceFAB } & 25 & 50 & 12.7 & 10 & 10 & 10 \\
& -25 & -50 & -12.7 & -10 & -10 & -10 \\
\hline \multirow{2}{*}{ Simulated PCR } & 25 & 50 & 16 & 28 & 30 & 24 \\
& -25 & -50 & -48 & -36 & -30 & -24 \\
\hline \multirow{2}{*}{ Real PCR } & 25 & 50 & 10 & 12 & 15 & 15 \\
& -25 & -50 & -30 & -18 & -15 & -15 \\
\hline
\end{tabular}

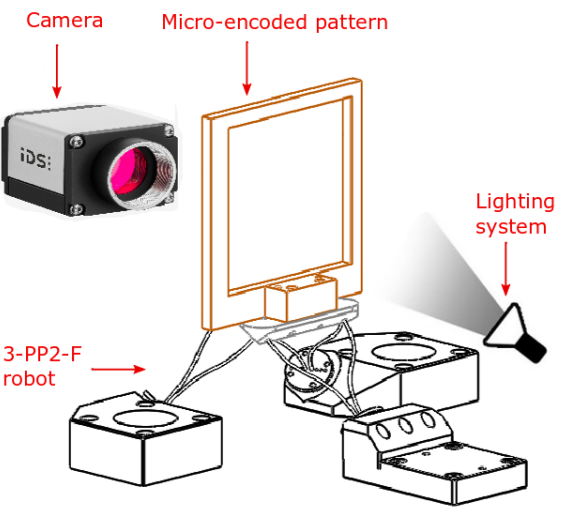

Fig. 6. Experimental setup showing the measurement system with the principal elements: the camera, the encoded pattern developed in [20], the PCR robot and the lighting system.

present in all the rotations ( $\mathrm{Rx}, \mathrm{Ry}$ and $\mathrm{Rz}$ ) showing the interest of replacing a spherical joint of the spaceFAB by slender flexible rods in the PCR.

\section{EXPERIMENTAL VALIDATION}

In this section, the experimental setup is described first and then the validation of the PCR is done by a comparison of three features: the workspace, the vertical stiffness and the position/orientation repeatability.

\section{A. Measurement system}

All the components are placed on an anti-vibration table to reduce the external noise affecting the measurement. The motion is captured by a vision measurement system that is composed of a camera IDS UI-3280CP-M-GL-R2, a LED backlight and an encoded pattern. The camera is configured with a frame rate of $13.35 \mathrm{fps}$. The measurement system can be seen in Fig. 6.

A phase-based method is used in order to get an accurate position and orientation measurement of an micro-encoded pattern attached to the robot platform. This algorithm together with a binary code can provide the absolute pose of the pattern down to $1 \mathrm{~nm}$ resolution in position and $4 \mu \mathrm{rad}$ in orientation over $10 \times 10 \mathrm{~cm}^{2}$ range. More details on this measuring method can be found in [20]. 


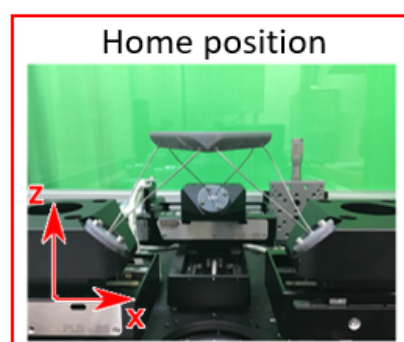

a)

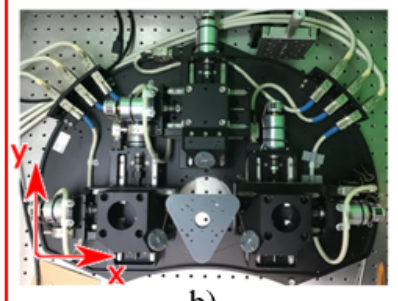

b)

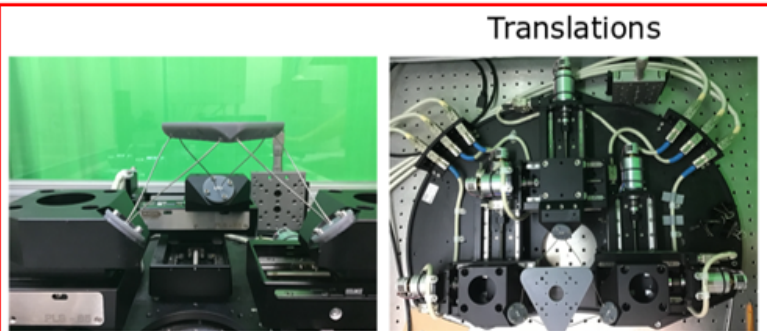

c)

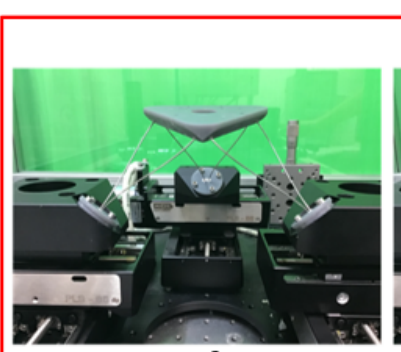

f) d)

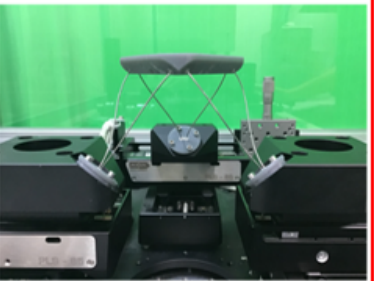

e)

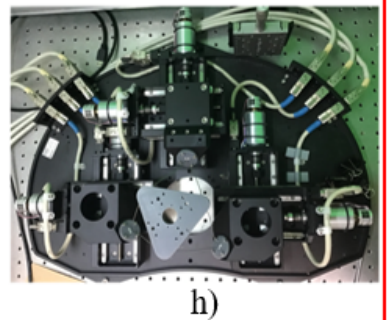

Fig. 7. Experimental results of the workspace around the 6-DoF provided by the PCR. a) $\mathrm{Tx}$ of $+25 \mathrm{~mm}$. b) $\mathrm{Ty}$ of $+50 \mathrm{~mm}$. c) Tz of $+12 \mathrm{~mm}$. d) $\mathrm{Rx}$ of $+10^{\circ}$. d) Ry of $+10^{\circ}$. d) $\mathrm{Rz}$ of $+10^{\circ}$.

\section{B. Workspace validation}

The procedure presented in IV-B has been used to evaluate the workspace of the PCR. The results are reported in Table III and shown in Fig. 7. The first two images ( $a$ and $b$ ) on the left are the home configurations of the PCR (side view and top view). Then c), d) and e) shows the translations in Tx, Ty and Tz respectively. Lastly f), g) and h) shows the rotations of Rx, Ry and Rz. These movements can be further appreciated on the video accompanying the paper.

These results validate that all the 6-DoF can be effectively be performed and that a larger workspace can be attained with the PCR than with the spaceFAB. The range of rotations reach $30^{\circ}$ in place of $20^{\circ}$. The range of translation along direction $\mathrm{Z}$ is $40 \mathrm{~mm}$ versus $25.4 \mathrm{~mm}$ for the spaceFab.

The experimental workspace is a little smaller than the simulated one. Indeed the maximum stress limit was not taken in consideration in simulation, so the rod deformations could exceed this value for extreme positions. Thus, to prevent any damage, the motions have been deliberately stopped before reaching the actuator stops.

\section{Experimental stiffness}

To estimate the vertical stiffness of the PCR, weights are added to the platform, keeping the actuators stationary at their neutral position. The smallest weight of $1.05 \mathrm{~kg}$ gives a stiffness of $216 \mathrm{~N} / \mathrm{mm}$ corresponding to a displacement of $47.7 \mu \mathrm{m}$.

The displacement is $104.5 \mu \mathrm{m}$ for $2.25 \mathrm{~kg}$ and $173.4 \mu \mathrm{m}$ for $3.6 \mathrm{~kg}$. This shows the non-linear force-displacement response of the PCR, meaning that it is less rigid as more load is added. This can be explained by the change of the shape of the rods.

\section{Experimental pose repeatability}

In this section, we followed the methodology established by the norm ISO 9283 [21] to evaluate the pose repeatability of the PCR. The pose repeatability quantifies the closeness of agreement between the attained poses after $n$ repeated visits to the same command pose in the same direction and is defined by its position and orientation repeatability. The position repeatability (PR) is defined as three times the standard deviation of the distance to the mean position of the platform. The orientation repeatability (OR) is defined as three times the standard deviation of angles.

Three conditions are established by the norm for this test: five points should be selected in such a way that all the workspace is attained, 30 cycles should be repeated in order to have enough data and a specific plan should be selected depending on the DoF tested. As Tx and Ty are assumed to be attained by the synchronized movement of all the actuators, our main focus is Tz. The repeatability of the robot is quantified using the vision-based reference system, and a warm-up of 200 cycles is launched before the recording of the images to reduce the drift introduced by the thermal expansion.

Once the images are recorded, the vision processing algorithm provides the positions and orientations of the platform. Fig. 8 shows the experimental results for the pose and orientation repeatability for the first position. Fig. 8(a) illustrates the distribution of the attained positions and the repeatability by the green circle of radius $P R=3.42 \mu \mathrm{m}$. The results for the orientations are in Fig. 8(b) where the green lines are the calculated limits from the mean (red line) with a value $O R= \pm 14.31 \mu \mathrm{rad}$.

To avoid any bias in the comparisons between the original articulated robot and the continuum one, the same proto- 

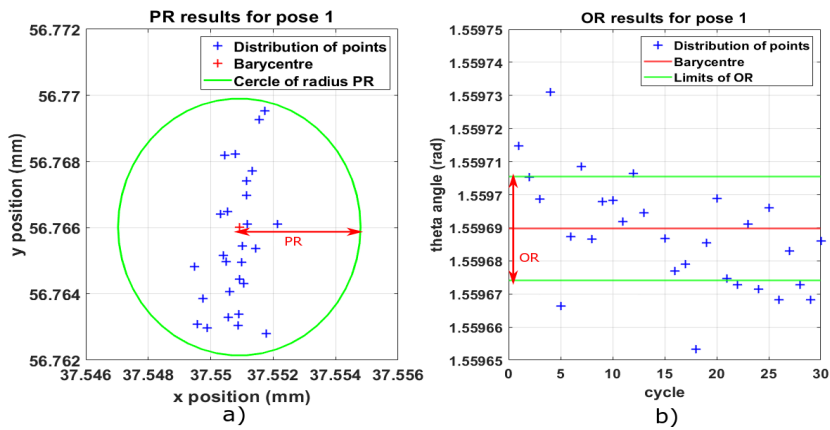

Fig. 8. Repeatability test results for pose $P_{1}$. In a) Position repeatability and in b) Orientation repeatability. The blue markers are the distribution of all the points, the red marker the coordinates of the barycentre and the green circle/lines represent the repeatability $P R$ and $O R$ respectively.

TABLE IV

EXPERIMENTAL REPEATABILITY RESULTS

\begin{tabular}{ccccc}
\hline & $\begin{array}{c}\text { Pose repeatability } \\
(\mu \mathrm{m})\end{array}$ & \multicolumn{2}{c}{$\begin{array}{c}\text { Orientation repeatability } \\
(\mu \mathrm{rad})\end{array}$} \\
\hline Position & spaceFAB & PCR & spaceFAB & PCR \\
\hline$P_{1}=[0,0,75]$ & 4.6 & 3.9 & 32.0 & 15.7 \\
$P_{2}=[5,0,80]$ & 4.6 & 2.8 & 27.9 & 11.2 \\
$P_{3}=[5,0,70]$ & 5.7 & 3.7 & 32.7 & 18.3 \\
$P_{4}=[-5,0,70]$ & 12.9 & 3.5 & 36.7 & 15.3 \\
$P_{5}=[-5,0,80]$ & 4.5 & 3.2 & 36.3 & 11.1 \\
\hline Mean & 6.46 & 3.42 & 33.12 & 14.31 \\
\hline
\end{tabular}

col has been used to evaluate the experimental pose and orientation repeatability of the spaceFAB. The results for both robots and the five positions are shown in Table IV, resulting in a PR value of $3.42 \mu \mathrm{m}$ and a $\mathrm{OR}$ value of $14.31 \mu \mathrm{rad}$ for the PCR. From these results, we can conclude that the position repeatability was reduced by $47 \%$ and the orientation repeatability by $57 \%$. This can be explained by the removal of the spherical and revolute joints but also by the constant stress in the structure that act as an anti backlash system on the axis composed of leadscrew motor.

\section{CONCLUSION}

In this paper, we proposed to transform a parallel articulated robot into a new parallel continuum one by replacing the links and the conventional passive joints by flexible rods. The workspace, stiffness and repeatability of the PCR were evaluated and compared to the articulated one.

The experiments show that the workspace of the PCR could be enlarged compared to the articulated robot, by $150 \%$ for rotations and by $157 \%$ along direction $\mathrm{Z}$. A vertical stiffness of $216 \mathrm{~N} / \mathrm{mm}$ at the reference position was measured. Furthermore, a repeatability of $P R=3.4 \mu \mathrm{m}$ in position and $O R=14.3 \mu \mathrm{rad}$ in orientation was demonstrated. The reached position and orientation repeatability are $47 \%$ and $57 \%$ better with the proposed structure than with the conventional articulated robot.

Finally, a reduction of $85 \%$ of the platform weight was also achieved due to the removal of the joints and the links, potentially allowing a future miniaturization of the robot.

\section{REFERENCES}

[1] C. E. Bryson and D. C. Rucker, "Toward parallel continuum manipulators," in IEEE International Conference on Robotics and Automation, Hong Kong, China, May 2014, pp. 778-785.

[2] C. Duriez, "Control of elastic soft robots based on real-time finite element method," in IEEE International Conference on Robotics and Automation, Karlsruhe, Germany, May 2013, pp. 3982-3987.

[3] A. L. Orekhov, C. B. Black, J. Till, S. Chung, and D. C. Rucker, "Analysis and Validation of a Teleoperated Surgical Parallel Continuum Manipulator," IEEE Robotics and Automation Letters, vol. 1, no. 2, pp. 828-835, July 2016.

[4] E. M. Young and K. J. Kuchenbecker, "Implementation of a 6DOF Parallel Continuum Manipulator for Delivering Fingertip Tactile Cues," IEEE Trans. Haptics, vol. 12, no. 3, pp. 295-306, 2019.

[5] F. J. Campa, M. Diez, D. Diaz-Caneja, and O. Altuzarra, "A 2 Dof Continuum Parallel Robot for Pick \& Place Collaborative Tasks," in Advances in Mechanism and Machine Science, vol. 73, 2019, pp. 1979-1988.

[6] B. Mauzé, R. Dahmouche, G. J. Laurent, A. N. André, P. Rougeot, P. Sandoz, and C. Clévy, "Nanometer precision with a planar parallel continuum robot," IEEE Robotics and Automation Letters, vol. 5, no. 3 , pp. 3806-3813, 2020.

[7] C. B. Black, J. Till, and D. C. Rucker, "Parallel Continuum Robots: Modeling, Analysis, and Actuation-Based Force Sensing," IEEE Transactions on Robotics, vol. 34, no. 1, pp. 29-47, Feb. 2018

[8] J. Till and D. C. Rucker, "Elastic Stability of Cosserat Rods and Parallel Continuum Robots," IEEE Transactions on Robotics, vol. 33 , no. 3, pp. 718-733, June 2017.

[9] J. Till, V. Aloi, and C. Rucker, "Real-time dynamics of soft and continuum robots based on cosserat rod models," The International Journal of Robotics Research, vol. 38, no. 6, pp. 723-746, 2019.

[10] O. Altuzarra, D. Caballero, F. J. Campa, and C. Pinto, "Position analysis in planar parallel continuum mechanisms," Mechanism and Machine Theory, vol. 132, pp. 13-29, Feb. 2019.

[11] B. Mauzé, G. J. Laurent, R. Dahmouche, and C. Clévy, "Micrometer positioning accuracy with a planar parallel continuum robot," Frontiers in Robotics and AI, vol. 8, p. 196, 2021.

[12] K. Nuelle, T. Sterneck, S. Lilge, D. Xiong, J. Burgner-Kahrs, and T. Ortmaier, "Modeling, Calibration, and Evaluation of a TendonActuated Planar Parallel Continuum Robot," IEEE Robotics and Automation Letters, vol. 5, no. 4, pp. 5811-5818, Oct. 2020.

[13] F. Tahmasebi and L.-W. Tsai, "Workspace and singularity analysis of a novel six-dof parallel minimanipulator," Applied Mechanisms and Robotics, vol. 1, 1994.

[14] M. Majid, Z. Huang, and Y. Yao, "Workspace analysis of a six-degrees of freedom, three-prismatic-prismatic-spheric-revolute parallel manipulator," International Journal of Advanced Manufacturing Technology, vol. 16, May 2000.

[15] S. H. Yang, Y. S. Kim, J. M. Yoo, and N. G. Dagalakis, "Microelectromechanical systems based Stewart platform with sub-nano resolution," Applied Physics Letters, 2012.

[16] N. Lobontiu, Compliant mechanisms: design of flexure hinges. CRC press, 2020.

[17] F. Faure, C. Duriez, H. Delingette, J. Allard, B. Gilles, S. Marchesseau, H. Talbot, H. Courtecuisse, G. Bousquet, I. Peterlik, and S. Cotin, "SOFA: A Multi-Model Framework for Interactive Physical Simulation," in Soft Tissue Biomechanical Modeling for Computer Assisted Surgery, vol. 11, June 2012, pp. 283-321.

[18] E. Coevoet, T. Morales-Bieze, F. Largilliere, Z. Zhang, M. Thieffry, M. Sanz-Lopez, B. Carrez, D. Marchal, O. Goury, J. Dequidt, and C. Duriez, "Software toolkit for modeling, simulation and control of soft robots," Advanced Robotics, pp. 1-26, Nov. 2017.

[19] T. Morales Bieze, A. Kruszewski, B. Carrez, and C. Duriez, "Design, implementation, and control of a deformable manipulator robot based on a compliant spine," The International Journal of Robotics Research, vol. 39, no. 14, pp. 1604-1619, Dec. 2020.

[20] A. N. André, P. Sandoz, B. Mauzé, M. Jacquot, and G. J. Laurent, "Sensing one nanometer over ten centimeters: A micro-encoded target for visual in-plane position measurement," IEEE/ASME Transactions on Mechatronics, 2020, in press.

[21] ISO 9283:1998, "Manipulating industrial robots - performance criteria and related test methods." International Organization for Standardization, Standard ISO 9283:1998, 1998. 